\title{
Diferencia en el incremento ponderal en el recién nacido de pretérmino y con peso bajo al nacimiento con método de madre canguro en comparación con incubadora
}

\author{
Patricia J. Ostía-Garza*, Lucero Moreno-Álvarez y Elga C. Reyes-Miranda \\ Departamento de Neonatología, Hospital Materno Perinatal "Mónica Pretelini Sáenz", Instituto de Salud del Estado de México. Toluca, México
}

\section{Resumen}

El bajo peso al nacer es un problema de salud pública en todo el mundo y se relaciona con una serie de consecuencias a corto y largo plazos. La técnica de madre canguro consiste en el manejo del recién nacidos de bajo peso al nacer o prematuros, basado en una triada consistente en calor, amor y leche materna. Objetivo: Determinar la diferencia en el incremento ponderal en recién nacidos de pretérmino y con peso bajo al nacimiento con método de madre canguro o la incubadora en pacientes hospitalizados en el Hospital Materno Perinatal "Mónica Pretelini Sáenz" de julio de 2017 a junio de 2018. Material y métodos: Se incluyó a 85 pacientes, en 45 de los cuales se aplicó la técnica de madre canguro y en 40 la atención con incubadora y se comparó el incremento de peso entre ambos. Resultados: Se confirmó que el $60 \%$ en el grupo de madre canguro alcanza el objetivo en tan sólo 10 días, mientras que el grupo de la incubadora lo hace en 20 días. Conclusión: Con la técnica de madre canguro el tiempo (días) para conseguir el peso objetivo de $1800 \mathrm{~g}$ es menor, ya que en el grupo de incubadora fue de $13.95 \pm 9.09$ días, en tanto que el grupo de madre canguro fue de $6.47 \pm 3.20$ días; se concluyó que, en promedio, el grupo de incubadora requiere el doble de tiempo que el grupo de madre canguro.

Palabras clave: Mamá canguro. Crecimiento. Recién nacido de pretérmino.

\section{Difference in growth in preterm and underweight infants at birth with kangaroo mother method vs incubator}

\section{Abstract}

Low birth weight remains a significant public health problem throughout the world and is associated with a series of short and long-term consequences. Preterm birth is the most frequent cause of neonatal mortality. The Kangaroo Mother technique consists in the ambulatory management of low birth weight or premature, based on a triad consisting of: Heat, Love and Maternal Milk. The benefits have been so noticeable that it has rapidly spread worldwide. Objective: To determine the difference in growth in preterm and underweight infants at birth with kangaroo mother method vs incubator in hospitalized patients at "Mónica Pretelini Sáenz" Hospital from July 2017 to June 2018. Material and methods: 85 patients were included 45 kangaroo mother technique was applied and 40 with traditional incubator management and the weight gain between both was compared. Results: It was confirmed that $60 \%$ of the patients in the group "kangaroo mom"reach the goal in just 10 days, while the "incubator group" does it in about 20 days. Significant significance in weight gain is confirmed with kangaroo te-

\section{Correspondencia:}

*Patricia J. Ostía-Garza

E-mail: patty_ostia@hotmail.com
Fecha de recepción: 03-05-2019

Fecha de aceptación: 16-08-2021 DOI: 10.24875/PER.19000026
Disponible en internet: 12-11-2021 Perinatol Reprod Hum. 2020;34(1):13-19

www.perinatologia.mx 0187-5337/@ 2021. Instituto Nacional de Perinatología Isidro Espinosa de los Reyes. Publicado por Permanyer. Este es un artículo open access bajo la licencia CC BY-NC-ND (http://creativecommons.org/licenses/by-nc-nd/4.0/). 
chnique being more than double in relation to incubator. Conclusion: it was demonstrated with the kangaroo mom technique the time (days) to reach the goal weight of $1800 \mathrm{~g}$ is lower, since in the incubator group it was $13.95 \pm 9.09$ days, while for the kangaroo mom group it was only $6.47 \pm 3.20$ days, concluding that on average, the incubator group requires twice as long as the "kangaroo mom" group.

Key words: Kangaroo mom. Newborn preterm. Growth.

\section{Introducción}

La situación crítica de niños prematuros y con bajo peso al nacer obliga a la medicina a buscar alternativas para solucionar el problema que desencadena, por ejemplo estancia prolongada en el hospital, infecciones cruzadas en aumento, ausencia de recursos de alta tecnología y mortalidad extremadamente alta'. Un aparato electromédico de gran utilidad usado durante muchos años es la incubadora, diseñada para proporcionar un ambiente térmico neutro que ayude al neonato a conservar su temperatura y humedad central normales, con un mínimo de consumo de oxígeno y gasto calórico. El incremento de recién nacidos prematuros, la ausencia de incubadoras y el manejo de éste por el ser humano son factores que intervienen como obstáculos o barreras para la consecución del objetivo. Al no satisfacer las demandas y carecer de condiciones óptimas para tratar a los recién nacidos prematuros y de bajo peso al nacer, es obligado realizar cambios y dejar a un lado la alta tecnología por recursos más económicos, pero no por eso menos eficientes, como el programa con la técnica de mamá canguro, que ofrece mejores y más rápidos resultados con un mínimo de recursos. Bajo los conceptos de "Cuidados canguro", "Atención canguro" o "Programa madre canguro" se ha amparado una cierta cantidad de intervenciones dirigidas primordialmente al recién nacido de pretérmino o de bajo peso al nacer ${ }^{2}$.

Los primeros en presentar este método fueron Rey y Martínez en el Hospital San Juan de Dios Bogotá, Colombia, en 1979, donde se desarrolló como alternativa a los cuidados de incubadora. Se consolidó durante los primeros 15 años bajo la coordinación de Martínez y Navarrete y se conoció como "Programa Madre Canguro". El grupo de investigación de la Fundación Canguro inició la evaluación científica del Programa Canguro del Instituto Materno Infantil de Bogotá en 1989 para luego crear en 1994 la Fundación Canguro, entidad sin ánimo de lucro, dedicada a la evaluación, mejoría y difusión del método de madre canguro (MMC) en el mundo ${ }^{3}$.

El programa se denominó así por considerar al recién nacido prematuro similar al marsupial que nace inmaduro y completa su maduración en contacto piel con piel con la madre.

La técnica de madre canguro consiste en el manejo ambulatorio de las niñas y los niños de bajo peso al nacer o prematuros, basado en una triada consistente en: calor, amor y leche materna. México ha hecho grandes esfuerzos por rescatar y asegurar la práctica de la lactancia materna en los niños y niñas sanos, si bien en los prematuros o de bajo peso este apoyo no ha sido tan constante, ya que a pesar de que varios hospitales lo trabajan en toda la República, no ha tenido continuidad ni uniformidad. El concepto de madre canguro permite un respeto y continuidad del vínculo que se establece entre la madre y el recién nacido desde la gestación, ya que el contacto piel con piel, además de mantener la temperatura del neonato, fortalece el instinto protector que tiene la madre hacia su hijo y asegura que lo estimule al tocarlo, hablarle, cantarle y, en ocasiones, sólo con el contacto la niña o niño escucha el corazón y la respiración, con las que ya está familiarizado desde su vida fetal ${ }^{4}$. Éstos son factores que proporcionan tranquilidad en el recién nacido, hacen que llore menos y que tenga vigilias tranquilas que lo ayuden a recuperar el peso ${ }^{5}$.

Inmediatamente al nacer, el neonato prematuro necesita apoyo para la transición del medio intrauterino al extrauterino. Durante las primeras $24 \mathrm{~h}$ de vida extrauterina pasa por un periodo vulnerable, debido a importantes ajustes fisiológicos que son necesario para su supervivencia. Durante esta fase inicial puede necesitar apoyo para establecer una respiración regular y mantener la temperatura corporal normal y cifras de glucosa para evitar situaciones potencialmente letales. Los métodos para la estabilización inicial incluyen ventilación mecánica, suministro de oxígeno, una vía intravenosa, nutrición parenteral, cunas o incubadoras para regulación de la temperatura. El programa de mamá canguro es una forma exitosa de cuidar a los recién nacidos estables de bajo peso al nacer, al colocarlos desnudos sobre el pecho de la madre 6 .

Dada la importancia que reviste el problema de bajo peso al nacimiento, la técnica de madre canguro resulta una alternativa segura, humana y respetuosa de la 
madre y el neonato, junto con un proceso de educación permanente a la familia que propicia la integración familiar del recién nacido en forma temprana ${ }^{7}$. Además propicia que otros miembros de la familia como el padre, los abuelos y hermanos se eduquen en el manejo canguro y con ello se atenúa el temor que sienten cuando tiene un recién nacido tan pequeño $0^{6,7}$. Se ha podido comprobar en hospitales de México donde se practica esta técnica que el tiempo de hospitalización necesario para la recuperación de estos recién nacidos es en promedio 18 días menor que en un manejo tradicional, lo que constituye un ahorro hospitalario y familiar, así como una menor morbilidad generada directamente por la hospitalización.

\section{Categorías del manejo madre canguro}

- Tardío: cuando el recién nacido está en cuidados intensivos e ingresa al programa después de siete días.

- Intermedio: el recién nacido ha completado los cuidados especiales y en menos de siete días inicia el programa.

- Temprano: el recién nacido se estabiliza fácilmente e inicia casi desde el nacimiento ${ }^{7}$.

El MMC repercute eficazmente en el control de la temperatura, la lactancia materna y el desarrollo de vínculos afectivos referidos a todos los neonatos, al margen de su entorno, peso, edad gestacional y situación clínica ${ }^{8}$. Los autores del MMC encontraron entre sus beneficios:

- Contacto piel a piel temprana, continua y prolongada entre la madre y el neonato.

- Lactancia materna exclusiva (en el caso ideal).

- Se inicia en el hospital y puede continuarse en el hogar.

- Los recién nacidos pequeños pueden recibir el alta en un plazo breve.

- Método amable y eficaz que evita la gran actividad que predomina por norma general en una sala de neonatología.

Los efectos del método sobre el aumento de peso son controvertidos. Algunos estudios que analizan el aumento de peso durante la hospitalización, como el de Ludington, et al., mostraron un aumento de peso de $19.72 \mathrm{~g} /$ día con técnica de mamá canguro en comparación con un incremento de peso de $10 \mathrm{~g} /$ día para niños que permanecieron en incubadoras; este análisis objetivo confirma una ganancia más significativa de peso en neonatos que recibieron $\mathrm{MMC}^{8}$.
El MMC equivale a la atención convencional (en incubadora) en lo que respecta a la seguridad y la protección térmica, facilita la lactancia materna, contribuye a la humanización de la atención neonatal, potencia el vínculo afectivo entre madre e hijo y es factible en cualquier entorno ${ }^{9}$.

En la llamada posición canguro, el niño se coloca en posición vertical sobre el pecho de la madre, entre sus senos y en decúbito ventral, con un contacto directo entre la piel de la madre y el hijo. La posición debe proveerse de forma continua (sin interrupciones) y prolongada, en condiciones ideales las 24 horas del día por tantos días como el niño lo requiera. El propósito de esta posición es que el recién nacido encuentre en la madre una fuente permanente de calor corporal, estimulación cinética y táctil, de tal forma que mantenga la vía respiratoria permeable. La posición estimula y favorece la lactancia materna. Además, el contacto íntimo y prolongado entre la madre y el hijo busca establecer o reforzar el vínculo biológico y afectivo sano que debe existir entre todo recién nacido y su madre y cuyo establecimiento se ve dificultado por la prematurez y enfermedad del neonato que lleva a una separación física madre-hijo ${ }^{10}$.

\section{Material y métodos}

Se realizó un estudio de tipo prospectivo, observacional, longitudinal y comparativo. La selección de los pacientes se asignó al azar para ambas técnicas. El grupo A incluyó la técnica de madre canguro, la cual consiste en el contacto piel con piel entre la madre y el recién nacido, de ser posible las $24 \mathrm{~h}$ del día; sin embargo, debido a lineamientos del servicio no fue posible hacerlo de esta manera, por lo que se aplicó de forma intermitente con intervalos de horario (de 09:00 h a 13:00 h y de 15:00 a 18:00 h), en estricta posición vertical, en medio del pecho de la madre, bajo su ropa; cabe mencionar que durante este periodo los pacientes fueron alimentados con leche materna o bien fórmulas lácteas. El grupo $B$ incorporó a aquellos pacientes atendidos con técnica convencional mediante incubadora de la marca Giraffe, la cual ajusta la temperatura al paciente.

Para llevar un seguimiento del incremento ponderal, los pacientes se pesaron diariamente por personal de enfermería asignado al área, con horarios de 06:00 a 8:00 am con básculas electrónicas neonatales de la marca RICE-LAKE, y el peso se registró en la hoja de enfermería correspondiente; de esta manera se efectuó después la vigilancia del incremento ponderal de 
Tabla 1. Análisis comparativo de la respuesta media de las semanas de gestación (SDG) en los grupos de estudio

\begin{tabular}{|l|c|c|c|c|c|c|c|}
\hline \multicolumn{7}{|c|}{ SDG } & \multicolumn{2}{c|}{ ANOVA } \\
\hline Grupo & $\mathrm{n}$ & Media & DE & Mínimo & Máximo & F & P \\
\hline Incubadora & 40 & 33.00 & 2.40 & 27.00 & 38.00 & 3.4649 & 0.066224 \\
\hline Mamá canguro & 45 & 32.02 & 2.44 & 27.00 & 37.00 & 38.00 & \\
\hline Todos & 85 & 32.48 & 2.45 & 27.00 & & \\
\hline
\end{tabular}

Fuente: Archivo Clínico del Hospital Materno-Perinatal Mónica Pretelini Sáenz.

los pacientes. Asimismo, se vigilaron y registraron estrechamente los signos vitales en ambos grupos por parte de enfermería.

Debe señalarse que durante el estudio no se presentó ningún efecto secundario en los pacientes tratados con la técnica de madre canguro, como hipotermia o alteraciones de los signos vitales. El estudio se realizó en los diferentes servicios de unidad de cuidados intensivos neonatales (internos y externos), unidad de cuidados intermedios neonatales y crecimiento y desarrollo. Los datos se anotaron en una hoja de recolección de datos diseñada para el estudio y análisis. Una vez obtenidos, los datos completos se interpretaron en gráficas, cuadros y descripciones. Se utilizó el programa estadístico Stadistica 10.0

\section{Resultados}

Durante el período de estudio se registraron 6,552 nacimientos y la incidencia de prematurez y bajo peso al nacer fue de $16 \%$ y $15 \%$, respectivamente. Se reunió a 85 pacientes que cumplieron con los criterios de inclusión, entre los cuales el criterio central fue ser de pretérmino o con peso $<1,800 \mathrm{~g}$. La composición de los grupos, por número de pacientes (Fig. 1), correspondió a 45 (52.9\%) niños en el grupo "mamá canguro" y $40(47.1 \%)$ en el grupo de niños atendidos en incubadora. Es un hecho que los niños que formaron los grupos de estudio, para su inclusión, cumplieron con peso $\leq 1,800 \mathrm{~g}$. Estos pesos se agruparon en tres categorías: 1) <1,000 g, 2) $1,001 \mathrm{~g}$ a $1,500 \mathrm{~g}$ y 3) $1,501 \mathrm{~g}$ a $1,800 \mathrm{~g}$.

El análisis estadístico reveló que el sexo está uniformemente distribuido en ambos grupos $(p=0.39327)$. Sólo 7 (8.2\%) pacientes presentaron un peso $<1,000 \mathrm{~g}$, 2 del grupo de incubadora y 5 del de mamá canguro (Tabla 1). En relación con las semanas de gestación se registraron en promedio $33.00 \pm 2.4$ SDG para el grupo de incubadora y de $32.02 \pm 2.44$ SDG para el grupo de

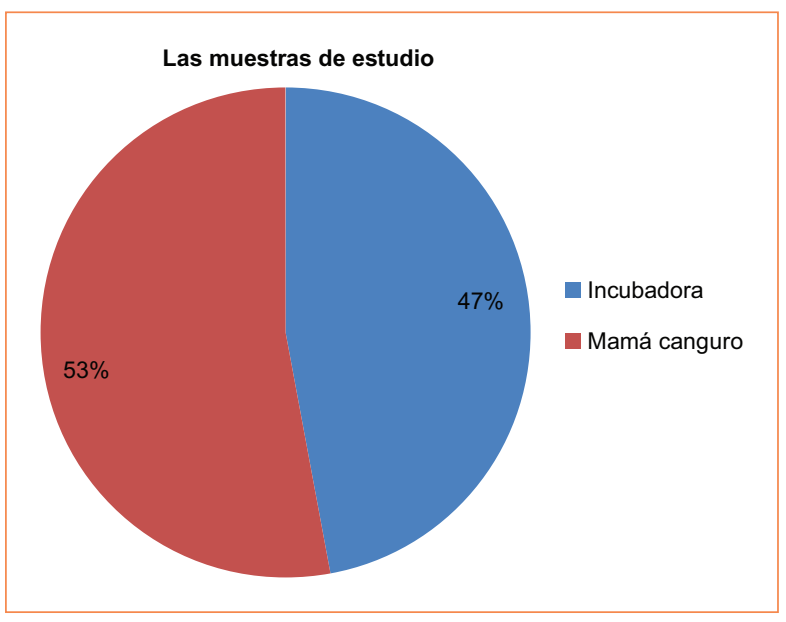

Figura 1. Grupos de estudio.

Fuente: Archivo Clínico del Hospital Materno-Perinatal Mónica Pretelini Sáenz.

mamá canguro. La prueba ANOVA de un factor reveló que estas media no alcanzaron una diferencia estadísticamente significativa ( $p=0.066224)$ (Fig. 2).

El análisis comparativo con ANOVA de un factor de la respuesta media señaló que el peso al nacimiento entre grupos fue de $1,416.08 \pm 254.70 \mathrm{~g}$ para el grupo de incubadora y de $1,334.62 \pm 301.84 \mathrm{~g}$ para el grupo "mamá canguro" ( $p=0.185377)$. El factor de la respuesta media del peso $(\mathrm{g})$ al ingreso al estudio entre grupos fue de $1,594.13 \pm 114.07 \mathrm{~g}$ para el grupo de incubadora y de $1,543.33 \pm 145.15 \mathrm{~g}$ para el grupo de "mamá canguro" ( $p=0.079077)$.

Se calculó el déficit de peso $(\mathrm{g})$ que presentaron los pacientes ante la objetivo de alcanzar los $1,800 \mathrm{~g}$ (Fig. 3). Este déficit se obtuvo con la diferencia entre $1,800 \mathrm{~g}$ con el peso al ingreso. El análisis comparativo de la respuesta media llevado a cabo con ANOVA de un factor del déficit de peso $(\mathrm{g})$ fue de $205.88 \pm 114.07 \mathrm{~g}$ para el grupo de incubadora y de $256 \pm 145.15 \mathrm{~g}$ para el grupo de "mamá canguro" (Tabla 2) $(p=0.049)$. 

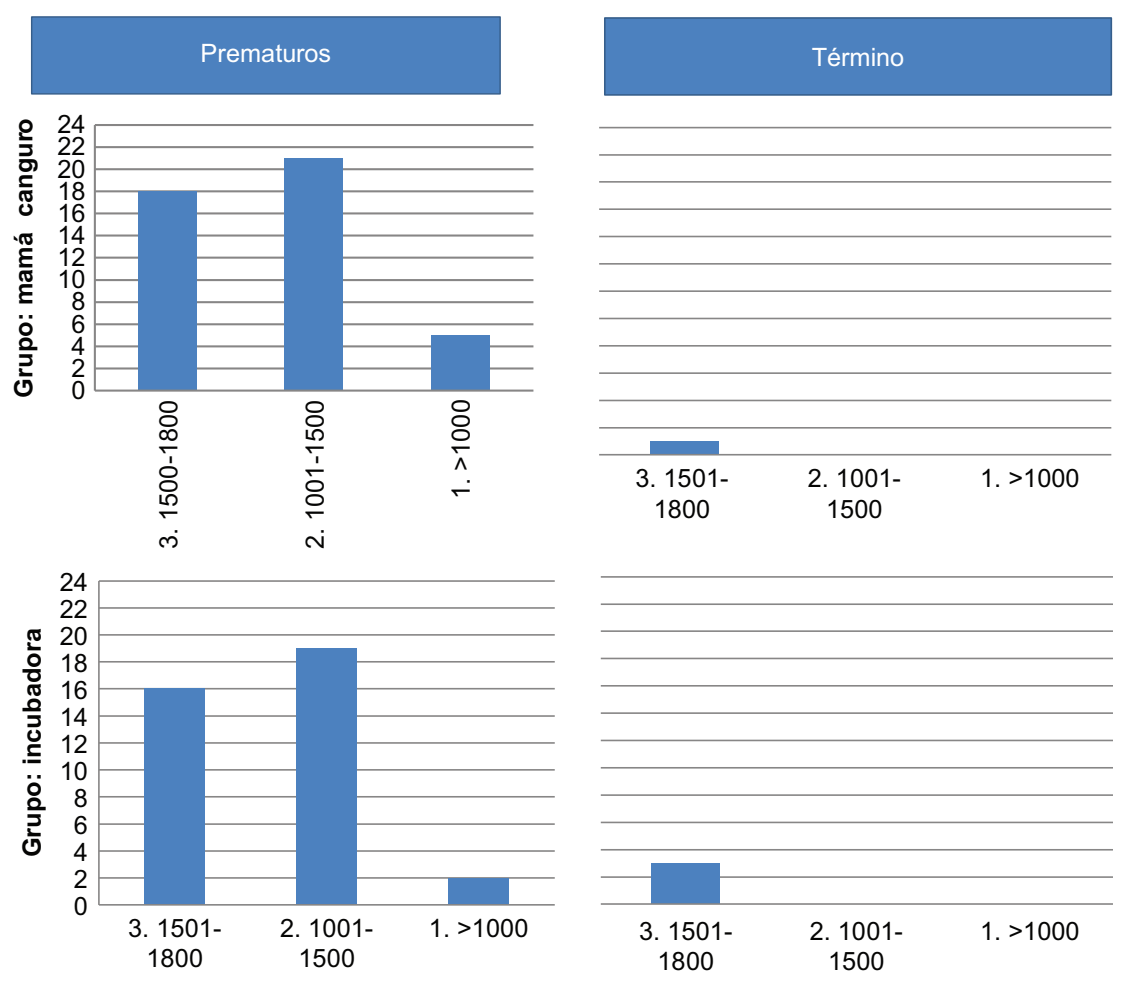

Figura 2. Distribución del peso de acuerdo con las semanas de gestación (de pretérmino o término) Fuente: Archivo Clínico del Hospital Materno-Perinatal Mónica Pretelini Sáenz.

Tabla 2. Comparación del déficit del peso (g) frente al objetivo de 1,800 g en los grupos bajo estudio

\begin{tabular}{|l|c|c|c|c|c|c|c|}
\hline \multicolumn{7}{|c|}{ Déficit $(\mathrm{g})$ del peso frente a 1,800 g } & \multicolumn{2}{c|}{ ANOVA } \\
\hline Grupo & $\mathrm{n}$ & Media & DE & Mínimo & Máximo & F & p \\
\hline Incubadora & 40 & 205.88 & 114.07 & 30.00 & 490.00 & 3.16106 & 0.049077 \\
\hline Mamá canguro & 45 & 256.67 & 145.15 & 80.00 & 600.00 & 600.00 & \\
\hline Todos & 85 & 232.76 & 133.14 & 30.00 & 6
\end{tabular}

Fuente: Archivo Clínico del Hospital Materno-Perinatal Mónica Pretelini Sáenz.

Se consideró como medida de eficacia los días de estancia hospitalaria desde el día de ingreso al estudio hasta el egreso del paciente. El tiempo (días) para el grupo de incubadora fue de $13.95 \pm 9.09$ días, mientras que para el grupo de mamá canguro fue de sólo 6.47 \pm 3.20 días. Se identificó que, en promedio, el grupo de incubadora requiere el doble de tiempo que el grupo de mamá canguro. La diferencia, de acuerdo con el ANOVA realizado, mostró una diferencia altamente significativa $(p=0.00002)$ (Fig. 4 y 5$)$.

De la misma forma se aplicó la prueba t de Student de los días para alcanzar el peso de 1,800 g, con 16.44 \pm 7.05 días para el grupo de mamá canguro, en tanto que para el grupo de incubadora fue de $38.35 \pm 7.29$ días con una importante significancia estadística, $p=0.000000000000000000000012$ (Tabla 3).

\section{Discusión}

Como señala el Manual de Atención Manejo Madre Canguro de Ruiz Matus, et al., ${ }^{5}$ se ha podido comprobar en hospitales donde se practica esta técnica que el tiempo de hospitalización necesario para la recuperación es en promedio 18 días menor que en una 
Tabla 3. Medidas de resumen estadístico y nivel de significancia estadística de la comparación de medias de los días para alcanzar la objetivo de 1,800 $\mathrm{g}$ en los grupos de estudio

\begin{tabular}{|l|c|c|c|c|c|c|c|}
\hline \multicolumn{9}{|c|}{ Días para alcanzar 1,800 g } & \multicolumn{3}{c|}{ ANOVA } \\
\hline Grupo & $\mathrm{n}$ & Media & DE & Mínimo & Máximo & F & 0.000002 \\
\hline Incubadora & 40 & 13.95 & 9.09 & 3.00 & 46.00 & 26.8118 & \\
\hline Mamá canguro & 45 & 6.47 & 3.20 & 2.00 & 17.00 & & \\
\hline Todos & 85 & 9.99 & 7.60 & 2.00 & 46.00 & \\
\hline
\end{tabular}

Fuente: Archivo Clínico del Hospital Materno-Perinatal Mónica Pretelini Sáenz

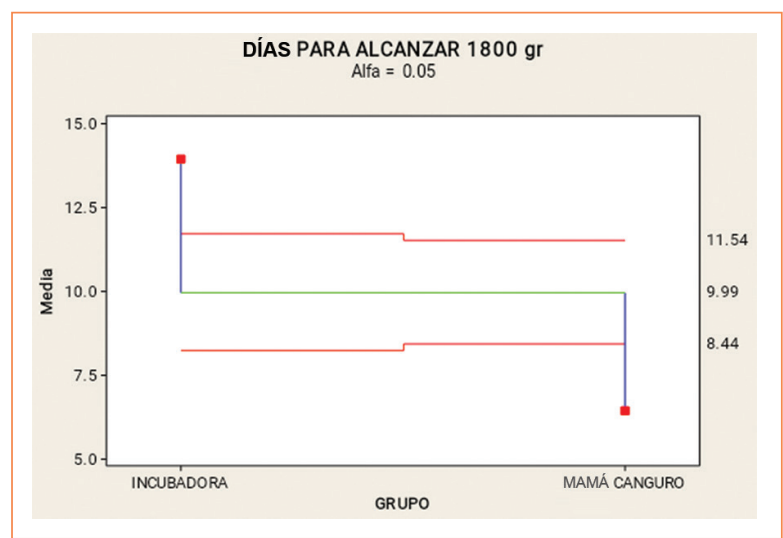

Figura 3. Efecto en el peso de acuerdo con el manejo asignado de los neonatos: mamá canguro o incubadora Fuente: Archivo Clínico del Hospital Materno-Perinatal Mónica Pretelini Sáenz.

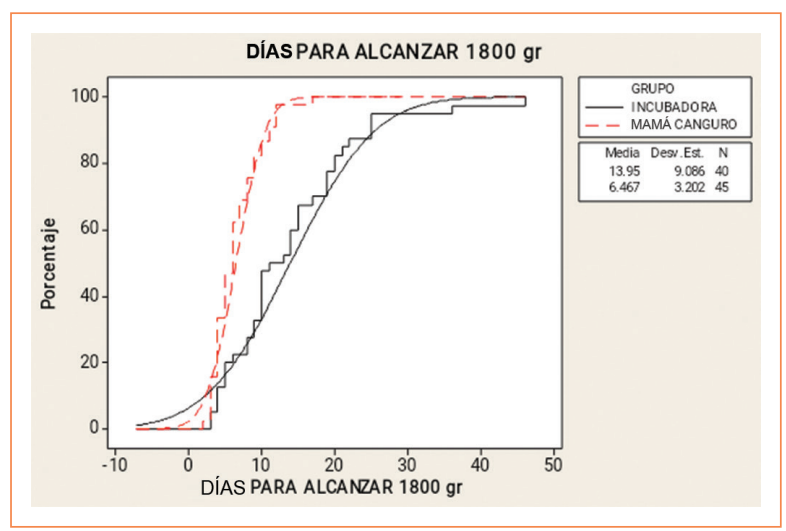

Figura 4. Análisis comparativo de acuerdo con el grupo de estudio de la frecuencia acumulada de los pacientes que alcanzan el objetivo de 1,800 g.

Fuente: Archivo Clínico del Hospital Materno-Perinatal Mónica Pretelini Sáenz.

atención tradicional; en este estudio se observó una disminución en días de estancia hospitalaria de siete días, lo que constituye un ahorro tanto hospitalario

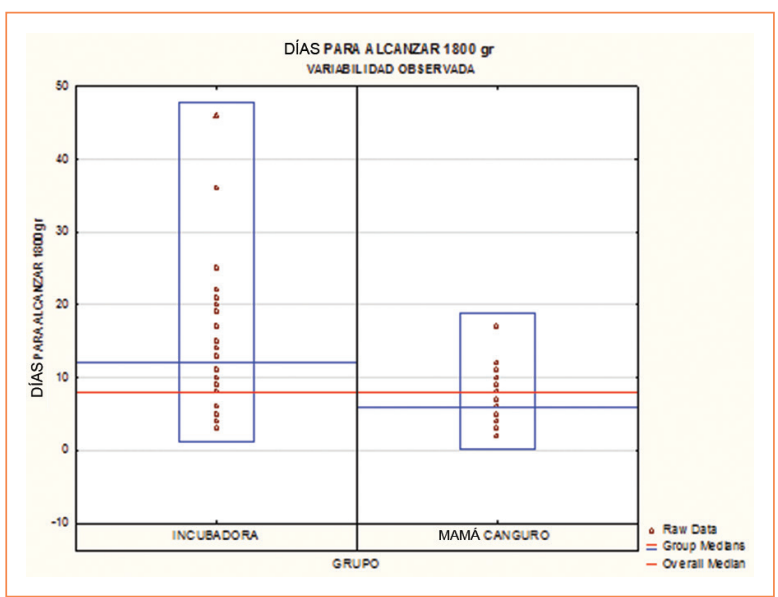

Figura 5. Descripción comparativa de la variabilidad observada de los días transcurridos para alcanzar el objetivo de los $1,800 \mathrm{~g}$.

Fuente: Archivo Clínico del Hospital Materno-Perinatal Mónica Pretelini Sáenz.

como familiar, así como una menor morbilidad generada directamente por la hospitalización.

\section{Conclusiones}

Se demostró que la técnica de mamá canguro produce un tiempo (días) para llegar al peso objetivo de $1800 \mathrm{~g}$ menor, ya que en el grupo de incubadora fue de $13.95 \pm 9.09$ días, mientras que para el grupo de mamá canguro fue de sólo $6.47 \pm 3.20$ días; en conclusión, el grupo de incubadora requiere en promedio el doble de tiempo que el grupo de "mamá canguro". El $60 \%$ de los pacientes en el grupo de "mamá canguro" alcanza el objetivo en apenas 10 días, en tanto que el "grupo de incubadora" lo hace en unos 20 días. Se confirma una significancia notoria en ganancia de peso con la técnica de mamá canguro, más del doble en relación con la incubadora. 


\section{Agradecimientos}

Los autores agradecen infinitamente a Dios, a los guerreros de UCINES que les permitieron ayudarles y a sus mamás que son unos pilares importantes para que este protocolo se llevara a cabo.

\section{Financiamiento}

Los autores declaran que no se recibió financiamiento alguno durante la realización de este proyecto.

\section{Conflicto de intereses}

Los autores declaran no tener conflicto de intereses en la publicación de este artículo.

\section{Responsabilidades éticas}

Protección de personas y animales. Los autores declaran que los procedimientos seguidos se conformaron a las normas éticas del comité de experimentación humana responsable y de acuerdo con la Asociación Médica Mundial y la Declaración de Helsinki.

Confidencialidad de los datos. Los autores declaran que han seguido los protocolos de su centro de trabajo sobre la publicación de datos de pacientes.

Derecho a la privacidad y consentimiento informado. Los autores han obtenido el consentimiento informado de los pacientes o sujetos referidos en el artículo. Este documento obra en poder del autor de correspondencia.

\section{Bibliografía}

1. Gallegos J, Reyes J, Gracinda G. La unidad neonatal y la participación de los padres en el cuidado del prematuro. Perinatol Reprod Hum. 2010;24(2):98-108.

2. Mares M, García A, Huerta A. Estudio de la técnica mamá canguro. Rev Hosp Gral Quebrada. 2002;1(1):16-19.

3. Rey ES, Martínez HG. Manejo racional del niño prematuro. Proceedings of the Conference I Curso de Medicina Fetal y Neonatal. Bogotá, Colombia, 1981;137:51.

4. Ruiz JG. Guía de práctica clínica basadas en evidencia para la óptima utilización del método madre canguro en el recién nacido pretermino y/o de bajo peso al nacer. Bogotá [actualizado 2007; citado 28 diciembre 2017]. Disponible en: http://www.quenoosseparen.info/documentos/ guias_evidencia.pdf

5. Ruiz Matus C, Martínez Peñafiel L. Manual de atención manejo madre canguro. 1ra ed. México: ISEM, 2006:1-37.

6. Almgren M. Benefits of skin-to-skin contact during the neonatal period: governed by epigenetic mechanisms. Genes \& Diseases 2018;5:24-26.

7. Angulo CE, García ME, Mancilla RJ, Estrada RI. Alimentación en el recién nacido. PAC de neonatología. Vol. 4. México: Intersistemas, 2016:1-61.

8. Ludington $\mathrm{S}$. Thirty years of kangaroo care science and practice. Neonatal netw. 2011;30(5):357-362.

9. Buil A, Carchon I, Apter G. Kangaroo supported diagonal flexion positioning: New insights into skin-to-skin contact for communication between mothers and very preterm infants. Arch Pediatr. 2016;23(1):913-920.

10. Penn S. Overcoming the barriers to using kangaroo care in neonatal settings. Nursing Children and Young People. 2015;27(5):22-27. 